\title{
Enhancing Petrographic Analysis Through Data Fusion
}

\author{
Nicholas Vito ${ }^{1}$, Connor Burt ${ }^{1}$ and Eric Goergen ${ }^{1}$ \\ 1. Thermo Fisher Scientific, Materials \& Structural Analysis, FEI Digital Rock Lab, Houston, TX, USA
}

Despite recent advances in characterization technology, there are still limitations in accuracy and throughput potential when characterizing textural relationships and diagenetic history of thin-bedded and lithic, clay-rich, and/or feldspar-rich reservoirs. These characteristics have large impacts on accurately assessing porosity and permeability profiles for upscaling into reservoir models. Several different methods, including scanning electron microscopy (QEMSCAN), mercury injection capillary pressure, and laser grain-size analysis are routinely used to quantify grain size, porosity, and mineralogy on thin-sections; however, each of these methods is associated with drawbacks on accuracy, time or both. One of those modalities alone is not adequate to describe most rock types. However, the continuous increases in computing power and the more common availability of advanced imaging technology and processing software has provided the opportunity for more accurate and statistically robust extraction of geological parameters from rock sample material. This presentation aims to show how fusing textural and mineralogical information derived from back-scattered electron (BSE), cathodolumenesce (CL) and energy-dispersive spectroscopy (EDS) improves rock characterization by obtaining measurements of grain size, shape, roundness, and composition in order to understand the diagenetic history

Using the BSE image, grains can easily be distinguished from pore space and organics at nanometer resolutions. Some minerals, such as a pyrite, can be easily identified from a silicate or carbonate matrix due to the relationship of BSE intensity with atomic number. However, many minerals have a substantially different chemistry but indistinguishable BSE singles due to similar mean atomic numbers. One example is to compare quartz $\left(\mathrm{SiO}_{2}\right)$ to albite $\left(\mathrm{NaAlSi}_{3} \mathrm{O}_{8}\right)$, which have mean atomic numbers of 10 and 10.03 respectively. The BSE image alone can provide a statistical approximation of grain size distribution, shape statistics, and roundness measurements. Automation of those measurements is possible, but many methods are not robust. Grain boundaries are often visible, but automated grain separation may be deterred by numerous internal grain fractures or bad approximations on non-spherical grains when using a chamfer-distance map and watershed separation algorithm. Despite these limitations, the wealth of data extracted from BSE images provides the contextual backdrop for the workflow provided in this presentation.

The CL image also has a resolution on the order of nanometers, but is more sensitive to chemistry and microstructure of the grains as the signal is recorded against the luminescence of the material caused by the bombarding electrons. The CL image specifically allows investigation of grain overgrowth which may be of the same material, a solid-solution with a different material, or a completely different material than the respective grain. Separation of the grain with the overgrowth and cement allows for better insight into the diagenetic history of the rock as shown in Figure 1.

The EDS image provides the best measurement on rock chemistry by matching elemental emission spectra from atomic relaxations to the acquired spectrum of the rock at every point in the image. Due to the interaction volume of the collected signal, the resolution is on the order of a micrometer. The relatively coarse resolution provides pixelated edges to fine-grained rocks or can completely miss 
detection. The mineral type can be assessed by specific ratios between the different detected elements.

The fusion process begins by having registered images of BSE, CL, and EDS acquired using automated tiling of all three modalities using automation software on an SEM outfitted with appropriate detectors. After acquisition, the images are exported to advanced image analysis software where the images are processed and correlated to determine measurements such as grain size distribution, shape, roundness, sorting, size of overgrowth, and porosity. Because of the EDS information, those measurements can be either global or specific to a mineral type as shown in Figure 2. The image-based workflow presented provides the opportunity to explore the combined imagery data in ways not easily accessed or as quantitative if explored as separate data. For example, the fusion workflow permits quantifying pore lining mineralogy and linking that information to specific pore types/textural location. Many spatial, textural and statistical relationships within rocks can be catalogued and evaluated using this technique. This presentation illustrates the nature of the workflow and provides examples of sandstones and mudrocks displaying varying amounts of diagenetic overprinting.
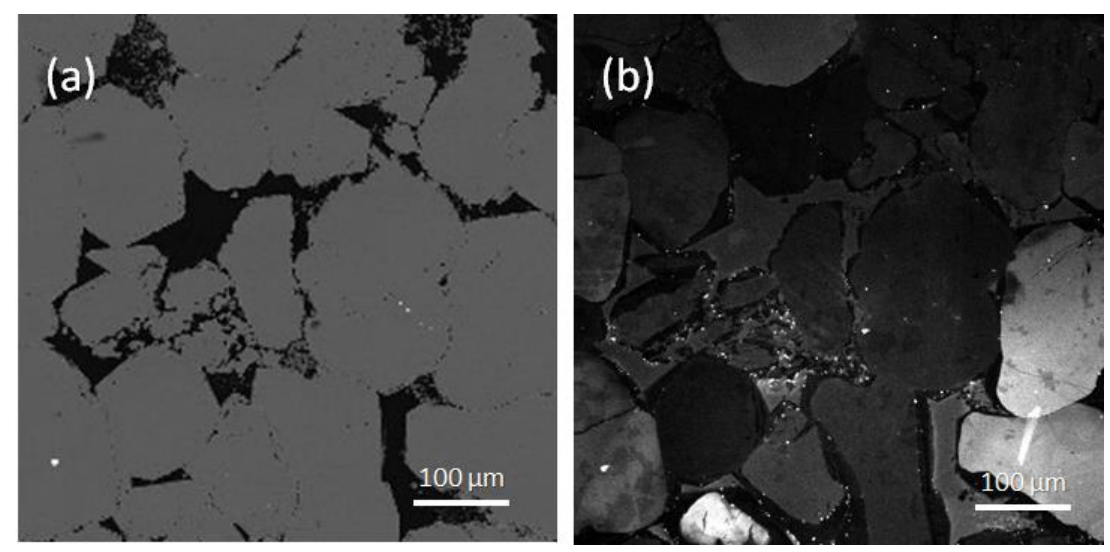

Figure 1. Comparison of the (a) BSE image with a (b) CL image acquired in a scanning electron microscope.
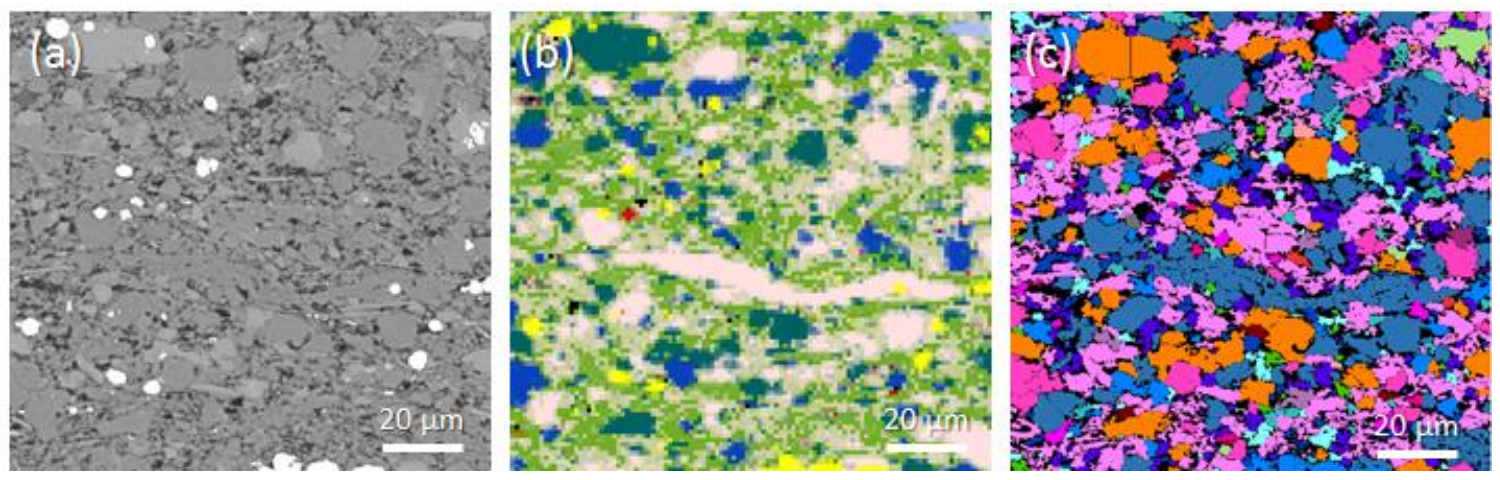

Figure 2. (a) BSE image, (b) mineralogy (EDS) map, and (c) example of data fusion of BSE and mineralogy map with chamfer based object separation. The colored regions in (c) correspond to the majority mineral in (b) within each separated grain. 